\title{
Enhancing folic acid metabolism suppresses defects associated with loss of Drosophila mitofusin
}

\author{
Juan Garrido-Maraver ${ }^{1}$, Ivana Celardo ${ }^{1}$, Ana C. Costa', Susann Lehmann', Samantha H. Y. Loh ${ }^{1}$ and L. Miguel Martins ${ }^{1}$
}

\begin{abstract}
Mutations in the mitochondrial GTPase mitofusin 2 (MFN2) cause Charcot-Marie-Tooth disease type 2 (CMT2A), a form of peripheral neuropathy that compromises axonal function. Mitofusins promote mitochondrial fusion and regulate mitochondrial dynamics. They are also reported to be involved in forming contacts between mitochondria and the endoplasmic reticulum. The fruit fly, Drosophila melanogaster, is a powerful tool to model human neurodegenerative diseases, including CMT2A. Here, we have downregulated the expression of the Drosophila mitofusin (dMfn RNAi) in adult flies and showed that this activates mitochondrial retrograde signalling and is associated with an upregulation of genes involved in folic acid (FA) metabolism. Additionally, we demonstrated that pharmacological and genetic interventions designed to increase the FA metabolism pathway suppresses the phenotype of the dMfn RNAi flies. We conclude that strategies to increase FA metabolism may ameliorate diseases, such as peripheral neuropathies, that are associated with loss of mitochondrial function. A video abstract for this article is available at https://youtu.be/fs1G-QRo6xl.
\end{abstract}

\section{Introduction}

Chartcot-Marie-Tooth (CMT) disease is the most common inherited neuromuscular disorder ${ }^{1}$, with no cure or treatment available. This peripheral neuropathy results from damage to neurons that transmit information from the brain and spinal cord to other parts of the body. Individuals with CMT suffer from muscle weakness and atrophy as well as mild sensory loss. There are two main types of CMT: type 1, where the damage occurs to myelin sheaths that surround the axons of neurons (CMT1) and type 2, where the damage is to the neuron itself (CMT2). Mutations in mitofusin 2 (MFN2) have been found to be one of the most common causes of CMT2, called CMT2a ${ }^{2}$.

\footnotetext{
Correspondence: Samantha H. Y. Loh (shyl2@mrc-tox.cam.ac.uk) or

L. Miguel Martins (martins.Imiguel@gmail.com)

${ }^{1}$ MRC Toxicology Unit, University of Cambridge, Lancaster Road, Leicester LE1 $9 \mathrm{HN}, \mathrm{UK}$

Edited by G. Melino

Enhancing folic acid metabolism restores mitochondrial function and is

neuroprotective following suppression of mitofusin.
}

Mitofusins are GTP-hydrolysing enzymes that promote mitochondrial fusion. They are important to ensure the dynamic balance between fusion and fission that determines mitochondria morphology. This dynamic balance also regulates mitochondrial health, since fission is crucial for recycling defective mitochondria through a process called mitophagy $\left(\right.$ reviewed in ${ }^{3}$ ).

Mitofusins can also act as organelle bridges. Mfn2 was reported to link mitochondria to the endoplasmic reticulum $(\mathrm{ER})^{4}$ and the downregulation of Drosophila mitofusin ( $d M f n$, also known as Marf) reduced contacts between the ER and mitochondria ${ }^{5}$.

The fruit fly, Drosophila melanogaster, is a powerful tool to study human neurodegenerative diseases (reviewed in $\left.^{6}\right)$. Moreover, pharmacological approaches can be used to test therapeutic candidates in flies. Drugs can be incorporated in the food and readily delivered. Since flies lack a stringent blood-brain barrier, these drugs can easily access the nervous system (reviewed in ${ }^{7}$ ).

Previously, it was reported that the ubiquitous downregulation of $d M f n$ in Drosophila is lethal at the larval

\section{(c) The Author(s) 2019}

(c) (i) Open Access This article is licensed under a Creative Commons Attribution 4.0 International License, which permits use, sharing, adaptation, distribution and reproduction cc) in any medium or format, as long as you give appropriate credit to the original author(s) and the source, provide a link to the Creative Commons license, and indicate if changes were made. The images or other third party material in this article are included in the article's Creative Commons license, unless indicated otherwise in a credit line to the material. If material is not included in the article's Creative Commons license and your intended use is not permitted by statutory regulation or exceeds the permitted use, you will need to obtain permission directly from the copyright holder. To view a copy of this license, visit http://creativecommons.org/licenses/by/4.0/. 
stage $^{8}$ and causes cellular toxicity by activating ER stress ${ }^{9}$. Here, we employed a strategy that enabled the generation of adult flies with ubiquitous downregulation of $d M f n$ and explored the consequences of its loss. We show the downregulation of $d M f n$ in adult flies compromises mitochondrial function and slows down the axonal transport of mitochondria in wing sensory neurons.

We used an exploratory analysis of the toxic consequences of downregulating $d M f n$ in adult flies. We uncovered an alteration of folic acid (FA) metabolism transcripts in flies with downregulated $d M f n$. We show that both dietary and genetic interventions to enhance FA metabolism partially supressed the defects observed in adult flies lacking $d M f n$. We conclude that strategies to enhance FA metabolism may prevent or delay the axonal defects in MFN2-linked CMT2a and other peripheral neuropathies associated with mitochondrial dysfunction.

\section{Results}

Reducing the expression of $d M f n$ compromises motor function in adult flies

The ubiquitous downregulation of $d M f n$ by RNAi using the tubulin-Gal4 driver is lethal at the larval stage ${ }^{8}$. By using an alternative ubiquitous driver, daughterless-Gal4 ( $d a \mathrm{Gal} 4$ ), we generated adult $d M f n$ RNAi flies where the $d M f n$ transcript was downregulated by $50 \%$ (Fig. 1a). This also reduced dMfn protein levels (Fig. 1b) and enabled approximately $50 \%$ of the adult flies to hatch (Fig. 1c). We then investigated the basal locomotor activity in $d M f n$ RNAi flies and found that the loss of $d M f n$ resulted in locomotor defects (Fig. 1d). Additionally, both the ubiquitous or pan-neuronal downregulation of $d M f n$ resulted in climbing defects (Fig. 1e).

\section{Downregulation of $d M f n$ expression results in a loss of mitochondrial cristae and reduced mitochondria axonal transport}

We next performed an analysis of mitochondria in $d M f n$ RNAi flies. A morphological analysis of the larval ventral nerve cord revealed fragmentation of mitochondria in the mechanosensory axons (Fig. 2a). Ultrastructural analysis of adult fly brains showed fragmented mitochondria cristae (Fig. 2b). To determine if the ultrastructural defects observed upon depletion of $d M f n$ were associated with a decrease in mitochondrial density (MD), we measured the levels of the mitochondrial matrix enzyme citrate synthase ${ }^{10}$, an indirect measurement of MD, in adult flies. Citrate synthase levels in $d M f n$ RNAi flies were not significantly altered compared to controls (Fig. 2c), indicating that the loss of mitochondrial cristae following depletion of $d M f n$ is not accompanied by a generalised loss of mitochondrial mass. Mitochondria are responsible for the generation of the majority of cellular ATP, a source of energy for axonal

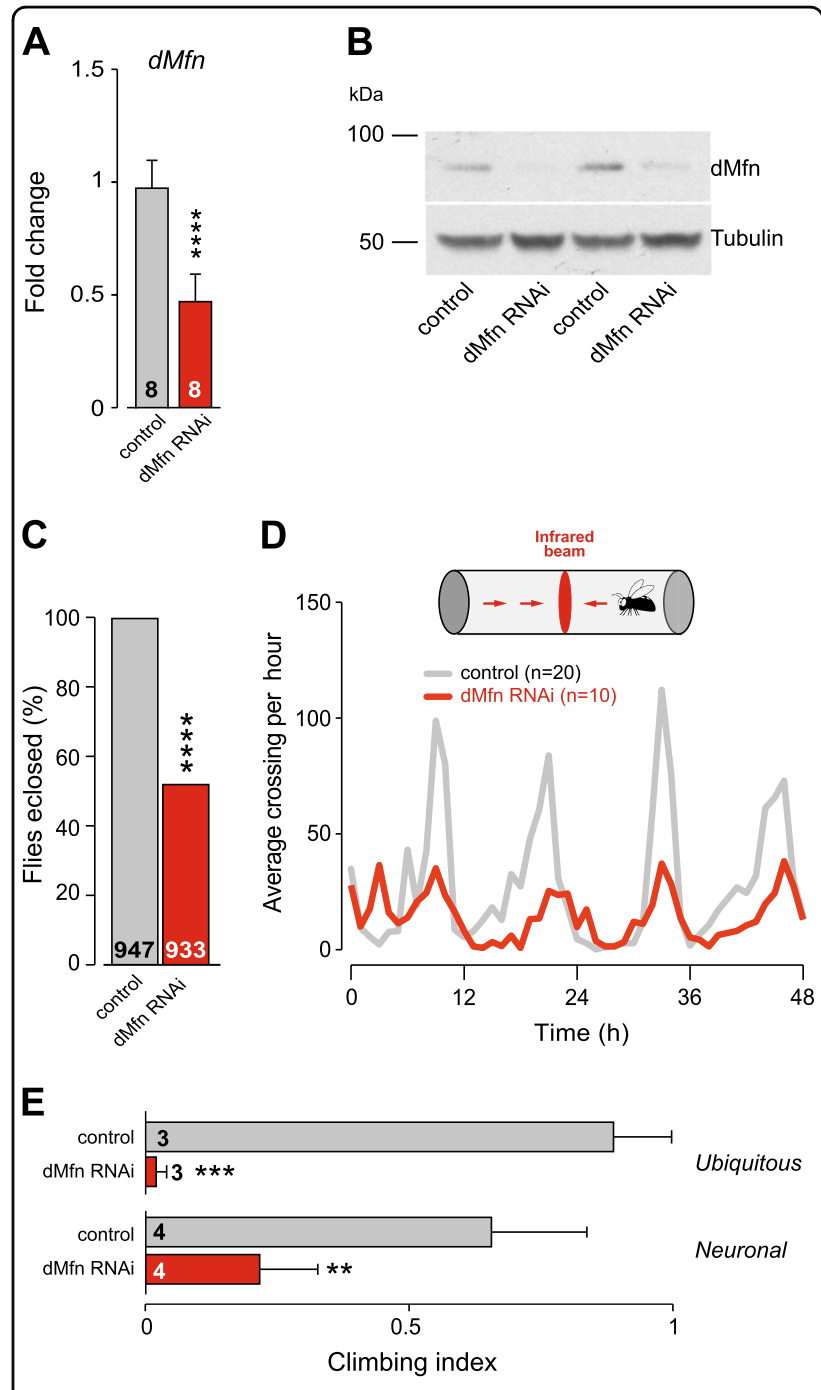

Fig. 1 In vivo suppression of $d M f$ causes motor impairment in adult flies. $\mathbf{a}$ and $\mathbf{b}$ RNAi-mediated suppression of $d M$ fn. $\mathbf{a}$ Expression levels of the $d M$ fn transcript were measured by real-time GPCR (mean $\pm \mathrm{SD}$; asterisks, two-tailed unpaired $t$-test compared to control). b Analysis of dMfn protein levels. Whole-fly lysates were analysed using the indicated antibodies. c Eclosion defects following RNAimediated suppression of dMfn (asterisks, chi-square two-tailed, 95\% confidence intervals). d Suppression of dMfn decreases locomotor activity. The number of flies tested is indicated for each genotype. e Motor impairment upon RNAi-mediated suppression of dMfn. Flies were tested using a standard climbing assay (mean \pm SD; asterisks, one-way ANOVA with Dunnett's multiple comparison test). Genotypes in (a-e (ubiquitous)): Control: $w_{;}+$; daGa/4/+, dMfn RNAi: $w$; dMfn RNAi/4; daGal4/+. (e (neuronal)): Control: w; elavGal4/4; +, dMfn RNAi: $w$; elavGal4/dMfn RNAi; +

mitochondrial transport (reviewed in $^{11}$ ). We also observed a significantly lower level of ATP in $d M f n$ RNAi flies compared to the control (Fig. 2d). Moreover, MFN2 mutations present in CMT2 patients cause defects in mitochondrial transport in cultured neurons ${ }^{12}$. We therefore assessed the effect of lowering $d M f n$ expression 


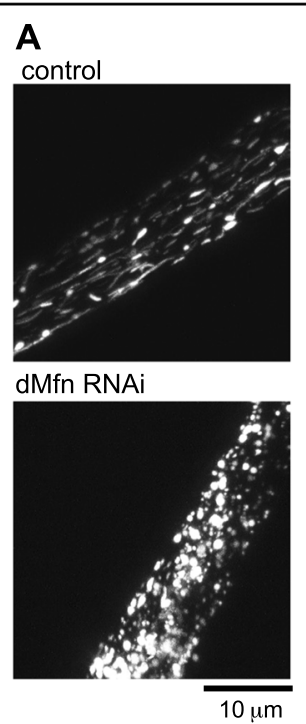

C

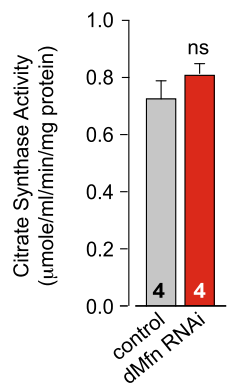

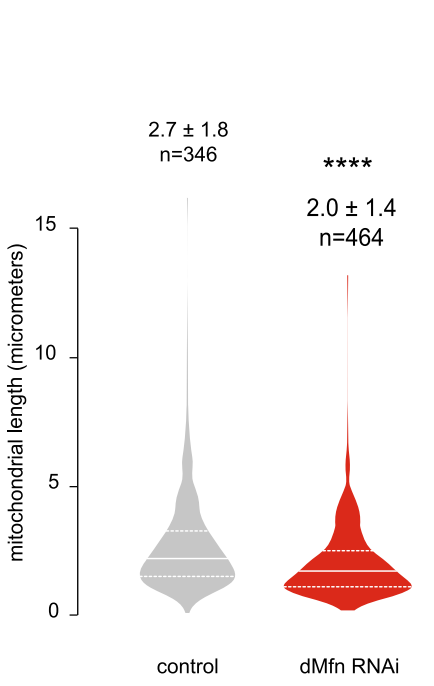

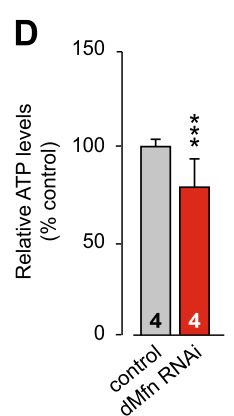

B

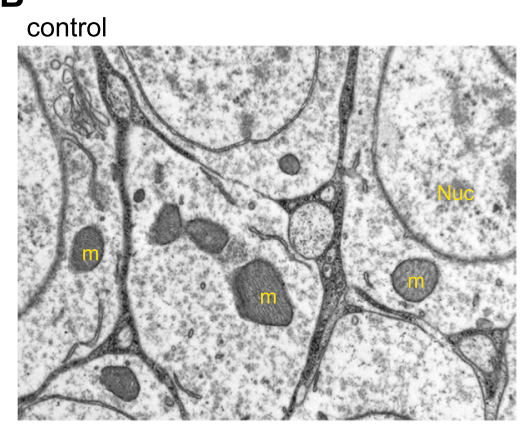

dMfn RNAi

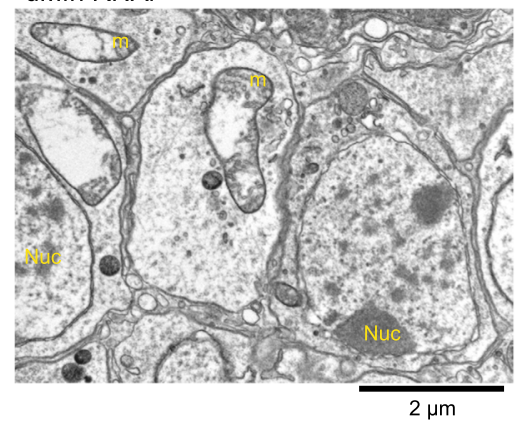

mitochondria with fragmented cristae (\%)

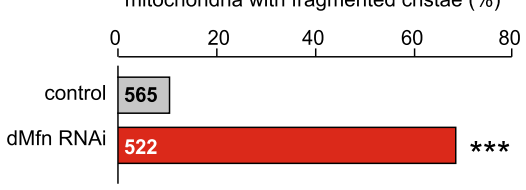

Fig. 2 Suppression of $d M$ m causes a loss of mitochondrial cristae density. a RNAi-mediated suppression of $d M$ mfn results in mitochondrial fragmentation. Confocal analysis of mitoGFP in the larval mechanosensory axons. The quantification of mitochondrial length is shown as a combined violin and box plot ( $p$ value, two-tailed unpaired $t$-test compared to control). $\mathbf{b}$ The knockdown of $d M f n$ causes defects in mitochondrial cristae. Ultrastructural analysis of the brains in adult dMfn RNAi flies ( $\mathrm{m}$, mitochondria; nu, cell nuclei). Asterisks, chi-square two-tailed, $95 \%$ confidence intervals. $\mathbf{c}$ The knockdown of $d M f n$ does not affect overall mitochondrial mass. Mitochondrial mass was assessed by measuring the activity of the mitochondrial matrix enzyme citrate synthase in adults ( $n s, p>0.05$, two-tailed unpaired $t$-test compared to control). $\mathbf{d}$ The knockdown of $d M f n$ causes a loss of ATP in adult flies (mean \pm SD, asterisks, two-tailed unpaired $t$-test compared to control). Genotypes in a Control: $w$; elavGal4/4; UASmitoGFP/+, dMfn RNAi: w; elavGal4/dMfn RNAi; UASmitoGFP/+. b-d Control: $w$; +; daGal4/+, dMfn RNAi: w; dMfn RNAi/+; daGal4/+

on the axonal transport of mitochondria to (retrograde) and away (anterograde) from the cell nucleus in wing sensory neurons (Vagnoni et al., 2016). We observed that lowering the expression of $d M f n$ results in decreased speeds for both anterograde and retrograde transport of mitochondria (Fig. 3).

We next focused on the functional status of mitochondria in adult flies with decreased expression of $d M f n$. High-resolution respirometry analysis revealed a significant decrease in respiration rates following suppression of $d M f n$ (Fig. 4a). Furthermore, we detected a loss of mitochondrial membrane potential $(\Delta \psi \mathrm{m})$ (Fig. $4 \mathrm{~b})$ and an increase in the levels of reactive oxygen species (ROS) (Fig. 4c, d) in the brains of adult flies with decreased expression of $d M f n$. These results, together with the loss of mitochondrial cristae integrity (Fig. 2b), suggest that the suppression of $d M f n$ does not affect the overall quantity of mitochondria but does compromise their function.

\section{Decreased expression of $d M f n$ is associated with the activation of ATF4-dependent targets associated with folic} acid metabolism

Debattisti and colleagues showed that loss of $d M f n$ activates ER stress ${ }^{9}$. We have recently demonstrated that ER stress linked to mitochondrial dysfunction causes the activation of dATF4, a transcription factor involved in cellular adaptation to stress ${ }^{13}$. To determine if a decrease in $d M f n$ could affect $d A T F 4$ expression, we measured the levels of activation of this transcription factor in $d M f n$ RNAi tissues using a reporter for $d A T F 4$ activation ${ }^{14}$. This analysis showed that the decreased expression of $d M f n$ led to a marked activation of $d A T F 4$ (Fig. 5a, b). The response to ER stress involves a 


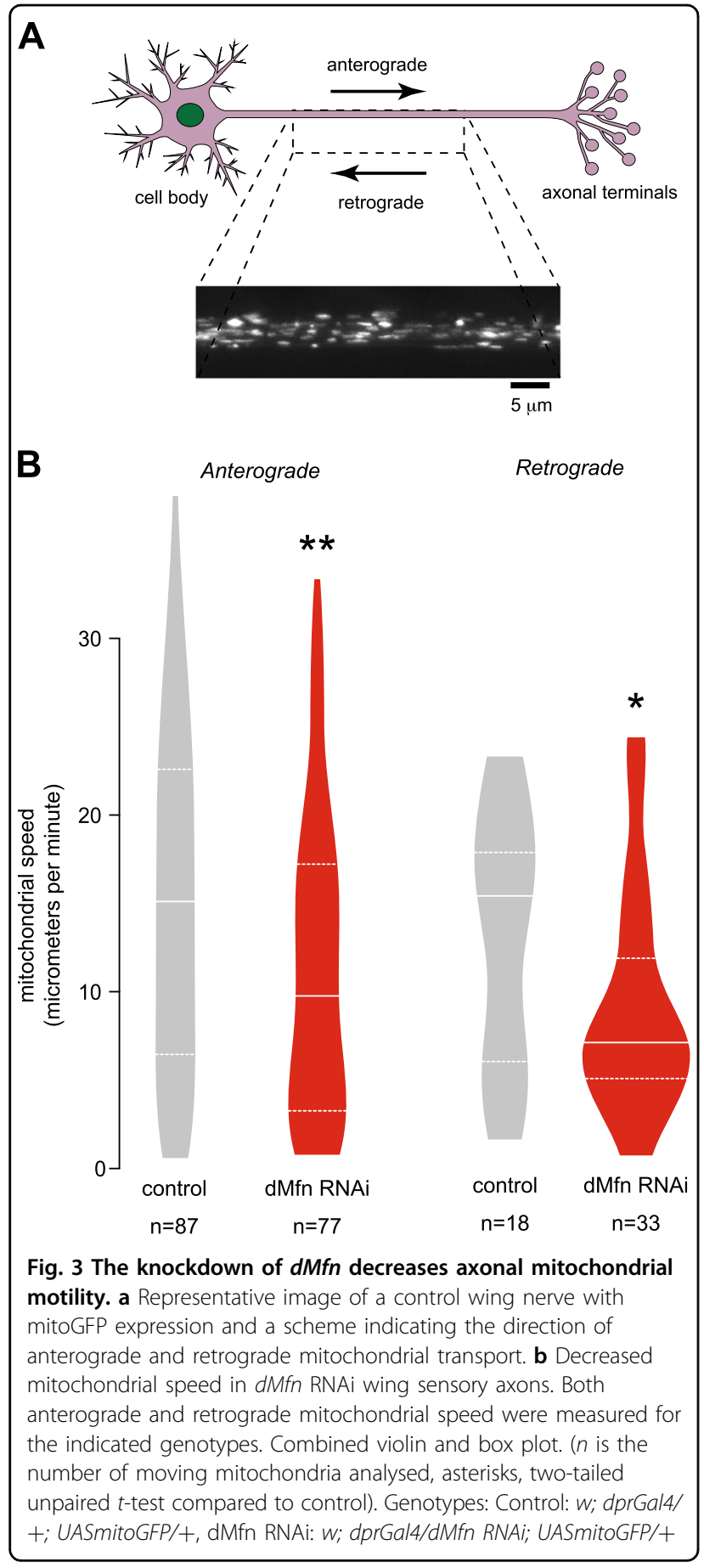

generalised decrease in protein translation mediated by PERK and a concomitant activation of ATF4-dependent transcriptional programmes designed to induce a cellular adaptation to stress (reviewed in ${ }^{15}$ ). To identify such transcriptional programmes in $d M f n$ RNAi flies, we took an unbiased approach by using microarray analysis. The analysis of transcriptional changes in $d M f n$ RNAi flies (Supplementary Table 1) showed a significant (FDR step-up $\leq 0.05)$ upregulation of 275 transcripts with fold-change $\geq 1.6$ (Supplementary Table 2). Next, a pathway analysis of the upregulated transcripts showed that the decrease in $d M f n$ expression causes an activation of genes involved in FA metabolism (Fig. 5c, d and Supplementary Table 3), including mitochondrial serine hydroxymethyl transferase (Shmt2) and mitochondrial NAD-dependent methylenetetrahydrofolate dehydrogenase $(N m d m c)$, two transcriptional targets of dATF4 that code for mitochondrial proteins ${ }^{13}$. The increased levels of Shmt2 and Nmdmc transcripts following $d M f n$ RNAi were confirmed in adult flies using real-time qPCR (Fig. 5e).

\section{A diet supplemented with folic acid suppresses mitochondrial dysfunction}

Folic acid has been historically used to treat anaemia during pregnancy. It functions as a carbon donor in metabolic reactions, including those involved in the synthesis of nucleotides from purine precursors (reviewed $\mathrm{in}^{16}$ ), and it has been shown to counteract mitochondrial dysfunction in the Drosophila central nervous system ${ }^{17}$.

Our data show that in the context of decreased levels of $d M f n$, flies attempt to compensate for mitochondrial stress by inducing the expression of FA metabolism genes. We therefore tested if enhancing this metabolic pathway by increasing the bioavailability of FA could rescue cellular defects associated with the loss of $d M f n$.

Maintaining $d M f n$ RNAi flies on an FA-supplemented diet during embryonic development did not alter mitochondrial fragmentation in larval mechanosensory axons (Fig. 6a) but was able to suppress loss of $\Delta \psi \mathrm{m}$ in the brains of adult flies (Fig. 6b). This FA-supplemented diet also suppressed the activation of dAtf4 (Fig. 6c, d) and rescued the eclosion defects of $d M f n$ RNAi flies (Fig. 6e). However, $d M f n$ RNAi flies raised on an FAsupplemented diet showed no significant difference in their lifespan (Fig. 6f) or locomotor activity (Fig. 6g) as compared to flies on a normal diet.

\section{Expression of $N m d m c$ rescues the phenotypes of $d M f n$ RNAi flies}

Genes involved in FA metabolism, such as Nmdmc, comprise a branch of mitochondrial retrograde signalling under the control of the dAtf4 transcription factor. We therefore tested the effect of enhancing the expression of $N m d m c$ in $d M f n$ RNAi flies. Increasing the expression of $N m d m c$ rescued mitochondrial function in neurons (Fig. 7a), suppressed the activation of dAtf4 (Fig. 7b) and decreased the eclosion defects of $d M f n$ RNAi adult flies (Fig. 7c). We also observed that the expression of $N m d m c$ increased the lifespan (Fig. 7d) and decreased the locomotor deficit (Fig. 7e) of $d M f n$ RNAi flies. Taken together, these results indicate that the expression of this 
A

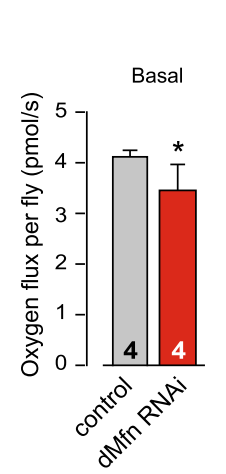

C

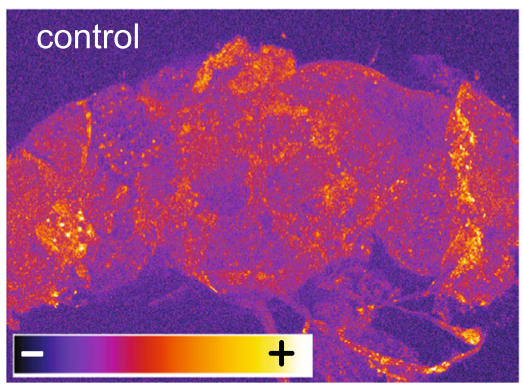

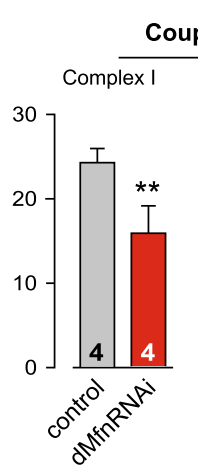

Coupled state 3

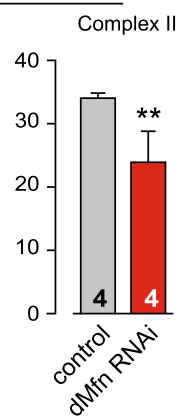

Uncoupled

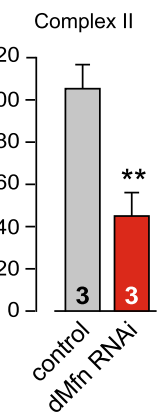

B

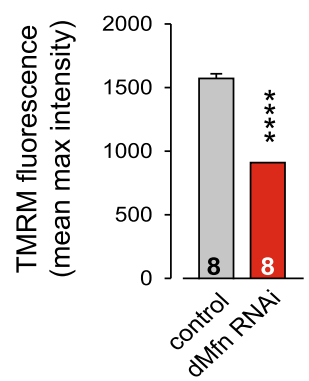

D

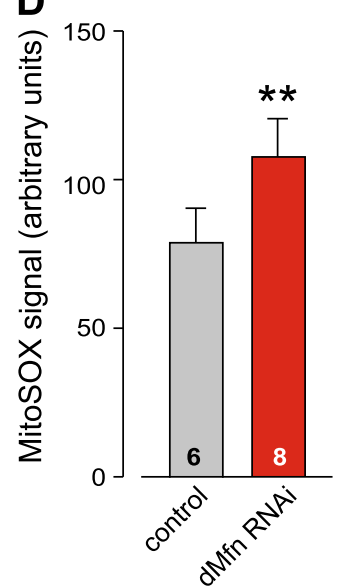

Fig. 4 The knockdown of $d M f n$ reduces mitochondria respiration and leads to an increase in reactive oxygen species. a Decreased respiration in $d M f n$ RNAi flies. Activity was measured by high-resolution respirometry in 3-day-old flies. Data are shown as the means \pm SD ( $n \geq 4$ in each genotype); asterisks, two-tailed unpaired t-test. $\mathbf{b}$ Loss of $\Delta \psi \mathrm{m}$ following RNAi-mediated suppression of $d M f n$. The data are shown as the mean \pm SD; asterisks, two-tailed unpaired t-test. $\mathbf{c}$ and $\mathbf{d}$ Increased mitochondrial ROS production in dMfn RNAi flies. c Representative images of adult fly brains stained with MitoSOX using false colour rendering. $\mathbf{d}$ Quantification of mitoSOX signal following the RNAi-mediated suppression of $d M f n$ (mean \pm SD; asterisks, two-tailed unpaired t-test). Genotypes: a-d Control: $w ;+$; daGal4/+, dMfn RNAi: w; dMfn RNAi/t; daGal4/+

gene, involved in FA metabolism, has a protective role upon loss of $d M f n$.

\section{Discussion}

Mitochondrial dynamics are coordinated by division and fusion. Fusion is coordinated by mitofusins, nuclear encoded mitochondrial transmembrane GTPases. Mitofusins act by promoting the fusion of the mitochondrial outer membrane (reviewed in ${ }^{18}$ ). Flies contain two genes coding for mitofusins, with $d M f n$ being the only mitofusin expressed in the somatic tissues of adults. Here, we show that the reduction of $d M f n$ in adult tissues leads to a fragmentation of mitochondrial cristae, compromising mitochondrial function and transport. Even though it was previously reported that the deletion of $M f n 2$ in cultured mouse cells increases mitochondrial mass ${ }^{19}$, we did not detect any alterations of mitochondrial mass after the in vivo depletion of $d M f n$. Our data also suggest that the observed fragmentation of mitochondrial cristae upon loss of $d M f n$ is not associated with loss of mitochondria since the overall mitochondrial mass is not altered.

The loss of $\Delta \psi \mathrm{m}$ and activation of $d A t f 4$ in flies depleted for $d M f n$ could be reversed with dietary supplementation with FA, suggesting that defective mitochondria can act as upstream activators of ER stress pathways. This backs our previous work showing that mitochondrial defects activate ER stress in pink1 or parkin mutant flies ${ }^{5}$.

The climbing defects and decreased lifespan observed in $d M f n$ RNAi flies could be improved by the expression of $N m d m c$, an enzyme involved in FA metabolism, but not by dietary supplementation with FA. Since the intracellular levels of FA intermediates were not measured in our study, we reason that flies lacking $d M f n$ might have a decreased food uptake or, alternatively, defects in the intestinal absorption of FA. This is in line with a previous study reporting that mitochondrial fragmentation triggered by the adult expression of Drp1, a protein that promotes 
A
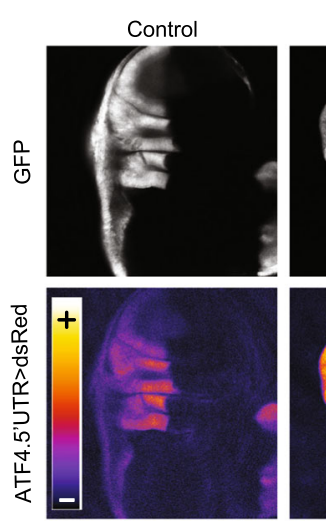

C

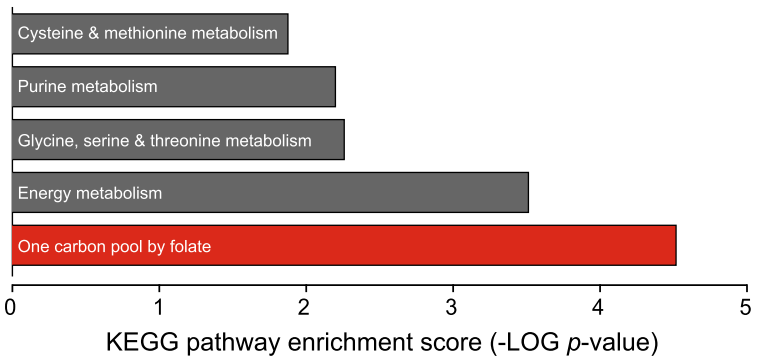

B
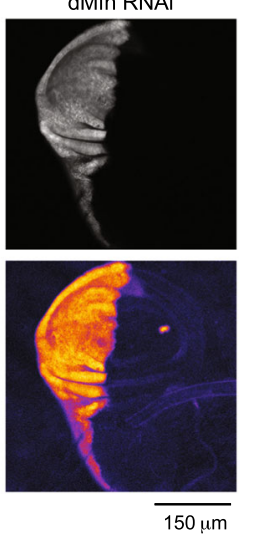

D

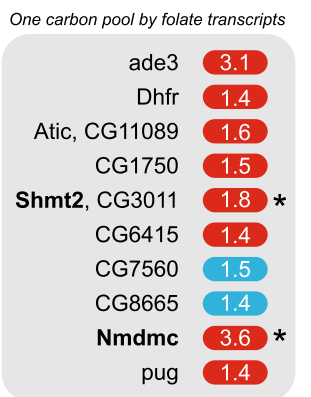

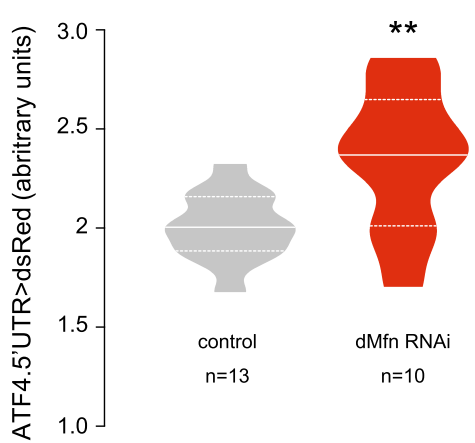

E

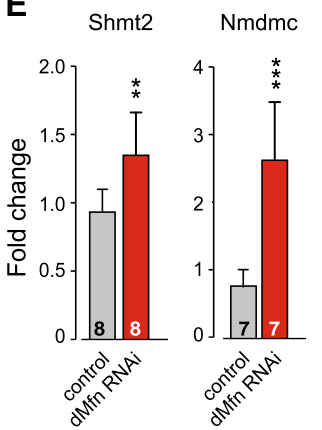

Fig. 5 The knockdown of $d M f n$ is associated with an enhanced expression of one-carbon metabolism transcripts. a and $\mathbf{b}$ The RNAimediated suppression of $d M f n$ activates $d A T F 4$. RNAi $d M f n$ was targeted at the posterior compartment of the larval wing disc using hhGal4 recombined with a GFP reporter. a Representative confocal images showing the enhanced in vivo expression of the ATF4.5'UTR $>$ dsRed reporter (lower right panel) following the RNAi-mediated suppression of $d M$ fn (posterior to the left and anterior to the right). $\mathbf{b}$ Quantitative analysis of dsRed fluorescence in the wing discs of the indicated genotypes. Combined violin and box plot (asterisks, two-tailed unpaired t-test). $\mathbf{c}$ and $\mathbf{d}$ Enhanced expression of one-carbon pool by folate metabolism transcripts following the RNAi-mediated suppression of $d M f n$. c Top pathways, comprising functionally related genes identified using Partek Pathway Analysis software are shown. P-values were calculated using Fisher's exact test. See also Supplementary Table 3. d Individual transcripts belonging to the one-carbon pool by folate metabolism enriched following the RNAi mediated suppression of $d M f n$. Red and blue correspond to, respectively, upregulated and downregulated transcripts above a statistical threshold (FDR $\leq 5 \%$ ). Asterisks indicate transcripts previously shown to be controlled by dATF4. e dMfn RNAi flies show an upregulation of mitochondrial Shmt2 and Nmdmc (mean \pm SD; asterisks, two-tailed unpaired t-test). Genotypes: a-b Control: $w$; ATF4.5' UTR > dsRed/4; hhGal4,UASGFP/+, dMfnRNAi: w; ATF4.5'UTR > dsRed/dMfnRNAi; hhGal4,UASGFP/+. c-e Control: $w$; +; daGal4/+, dMfn RNAi: w; dMfn RNAi/+; daGal4/+

mitochondrial fission, compromises the integrity of the fly intestinal barrier ${ }^{20}$. This, in turn, might explain why our pharmacological intervention was less efficient than a genetic enhancement approach.

A therapeutic approach to treat Charcot-Marie-Tooth disease type 2 by manipulating mitofusin conformations has been proposed recently ${ }^{21}$. Our data show that enhancement of FA metabolism might also be a viable approach to treat Charcot-Marie-Tooth disease type 2 and other diseases linked to mitochondrial dysfunction.

\section{Methods}

\section{Genetics and Drosophila strains}

Fly stocks and crosses were maintained on standard cornmeal agar media at $25^{\circ} \mathrm{C}$. The strains used were daGAL4, $w^{1118}$, elavGAL4, UASmitoGFP (Bloomington Stock Centre), RNAi line $d M f n$ (ID: 105261,Vienna
Drosophila RNAi Center), UAS Nmdmc as previously described $^{13}$, hhGal4, UASGFP (kind gift from H. Steller, Rockefeller University, New York, USA), $d p r$ Gal4 (kind gift from S. Bullocks, MRC Laboratory of Molecular Biology, Cambridge, UK) and $d A T F 4.5^{\prime} U T R>d s$ Red reporter (kind gift from K. Kang and M-J. Kang, University of Ulsan College of Medicine, Seoul, Republic of Korea). All experiments on adult flies were performed using only males.

\section{RNA extraction and quantitative real-time RT-PCR}

Total RNA was extracted using TRIzol (Ambion) and quantified by spectrophotometric analysis. Quantitative real-time PCR with reverse transcription (qRT-PCR) was performed on a real-time cycler (Applied Biosystems 7500 Fast Real-Time PCR Systems) using the SensiFAST SYBR Lo-ROX one-Step Kit (Bioline). Gene-specific primers were obtained from QIAGEN (QuantiTect Primer Assays) 


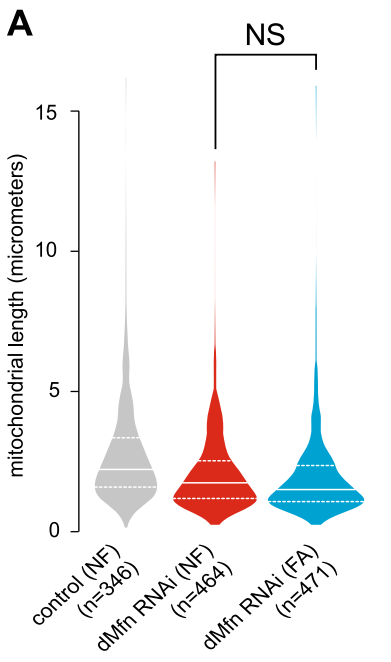

D

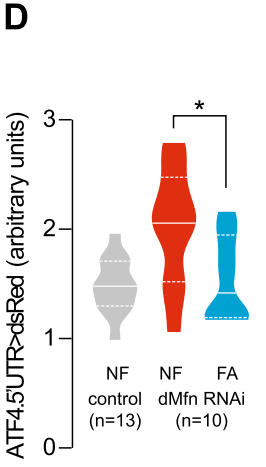

$\mathbf{E}$
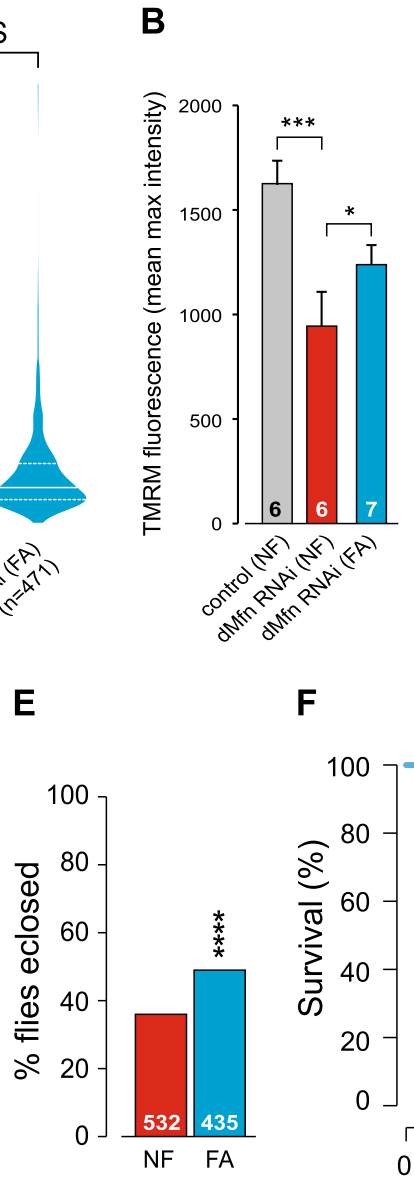

$\mathbf{F}$

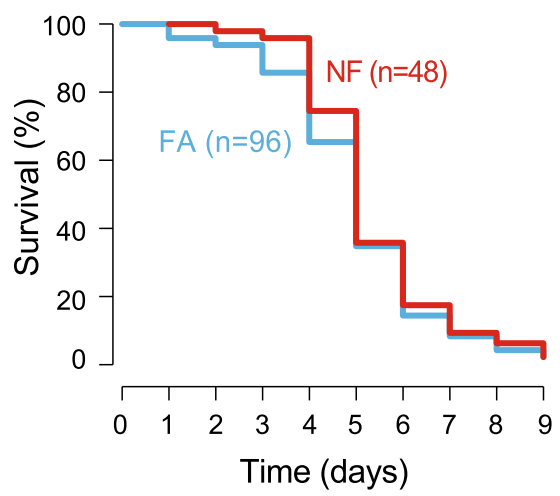

C
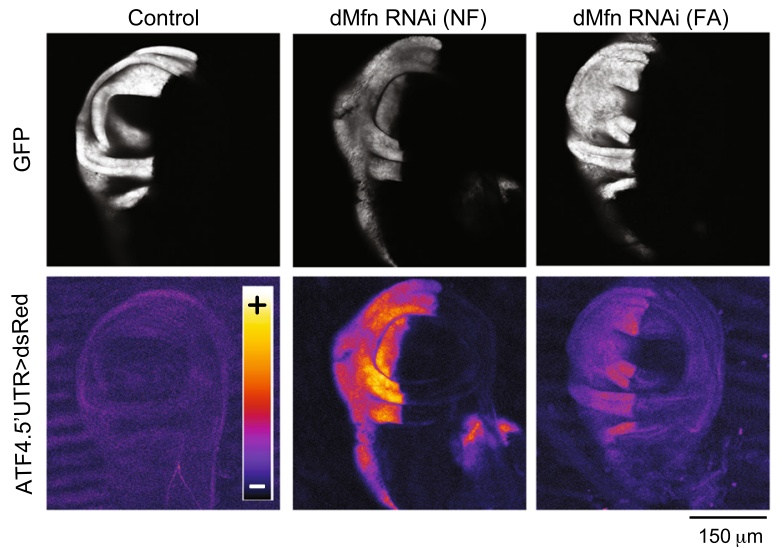

G

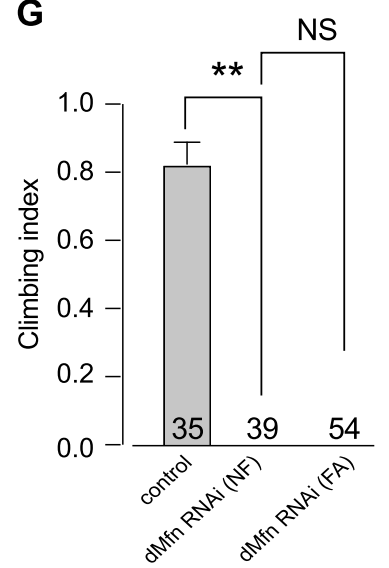

Fig. 6 Dietary supplementation with FA suppresses defects associated with RNAi-mediated suppression of $d$ Mfn. Dietary supplementation with FA does not affect mitochondrial fragmentation in dMfn RNAi flies (a) but partially reverses the loss of $\Delta \psi m$ (b) (mean \pm SD; asterisks, one-way ANOVA with Bonferroni's multiple comparison test). c, d Dietary supplementation with FA suppresses the increased expression of $d A T F 4$ in dMfn RNAi flies. Representative confocal images and quantitative analysis of dsRed fluorescence in the indicated wing discs are shown (asterisk, two-tailed unpaired $t$-test). e Decreased eclosion defects in dMfn RNAi flies, following dietary supplementation with FA (asterisks, chi-square two-tailed, 95\% confidence intervals). f An FA-supplemented diet does not modify lifespan upon RNAi-mediated suppression of $d M f n$. Fly viability was scored over a period of 10 days. $\mathbf{g}$ An FA supplemented diet does not rescue the locomotor defects of $d M$ fn RNAi flies. Flies were tested using a standard climbing assay (mean \pm SD; asterisks, one-way ANOVA with Dunnett's multiple comparison test). NF normal food; FA folic acid supplemented food. Genotypes: (a) Control: w; elavGal4/+; UASmitoGFP/+, dMfn RNAi: w; elavGal4/dMfn RNAi; UASmitoGFP/+, c-d Control: w; ATF4 5' UTR DsRed/+; hhGal4,UASGFP/+, dMfn RNAi: $w$; ATF4.5' UTR > dsRed/dMfn RNAi; hhGal4,UASGFP/+, e-f w; dMfn RNAi/+; daGal4/+ and (b and g) Control: w; +; daGal4/+, dMfn RNAi: w; dMfn RNAi/+; daGal4/+. Datasets in (a), labelled control (NF) and dMfn RNAi (NF) are also used in Fig. 2a. Dataset in (b) labelled dMfn RNAi (NF) is also used in Fig. 7a, labelled dMfn RNAi. Dataset in (d) labelled control (NF) and dMfn RNAi (NF) are also used in Fig. 5b

for the following genes: $d M f n$ (QT00499205), Nmdmc (dm: QT00503153), Shmt2 (dm: QT00498904). Genespecific primer rp49 (forward, TGTCCTTCCAGCTT CAAGATGACCATC; reverse, CTTGGGCTTGCGCCA TTTGTG) was obtained from Sigma and used as a housekeeping gene.

\section{Protein extraction and western blotting}

Protein extracts from whole flies were prepared by grinding flies in lysis buffer $(100 \mathrm{mM} \mathrm{KCl}, 20 \mathrm{mM}$ Hepes at $\mathrm{pH} 7.5,5 \%(\mathrm{v} / \mathrm{v})$ glycerol, $10 \mathrm{mM}$ EDTA, $0.1 \%(\mathrm{v} / \mathrm{v})$
Triton X-100, $10 \mathrm{mM}$ DTT, $(1 \mu \mathrm{g} / \mathrm{mL}$ leupeptin, $1 \mu \mathrm{g} / \mathrm{mL}$ antipain, $1 \mu \mathrm{g} / \mathrm{mL}$ chymostatin and $1 \mu \mathrm{g} / \mathrm{mL}$ pepstatin). The suspensions were cleared by centrifugation at $21,000 \times g$ for $10 \mathrm{~min}$ at $4{ }^{\circ} \mathrm{C}$ and protein concentrations of the supernatants were measured using the Bradford assay (Bio-Rad). All supernatants were mixed with $4 \times$ LDS loading buffer. For SDS-PAGE, equivalent amounts of proteins were resolved on 10\% Precast Gels (Invitrogen) and transferred onto PVDF membranes (Millipore). The membranes were blocked in TBS $(0.15 \mathrm{M} \mathrm{NaCl}$ and 10 $\mathrm{mM}$ Tris- $\mathrm{HCl}, \mathrm{pH}$ 7.5) containing $10 \%(\mathrm{w} / \mathrm{v})$ dried non-fat 


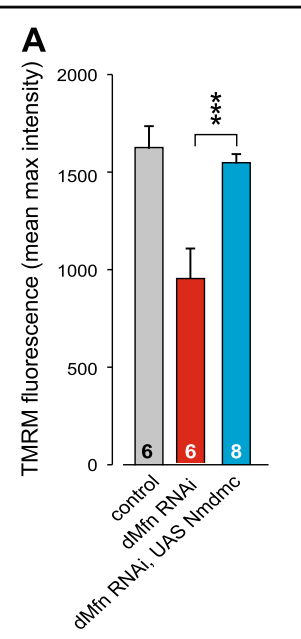

B

D

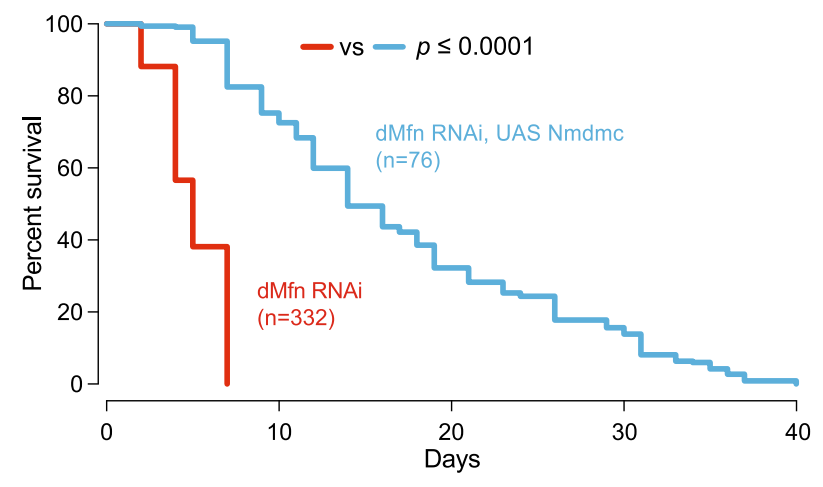

C
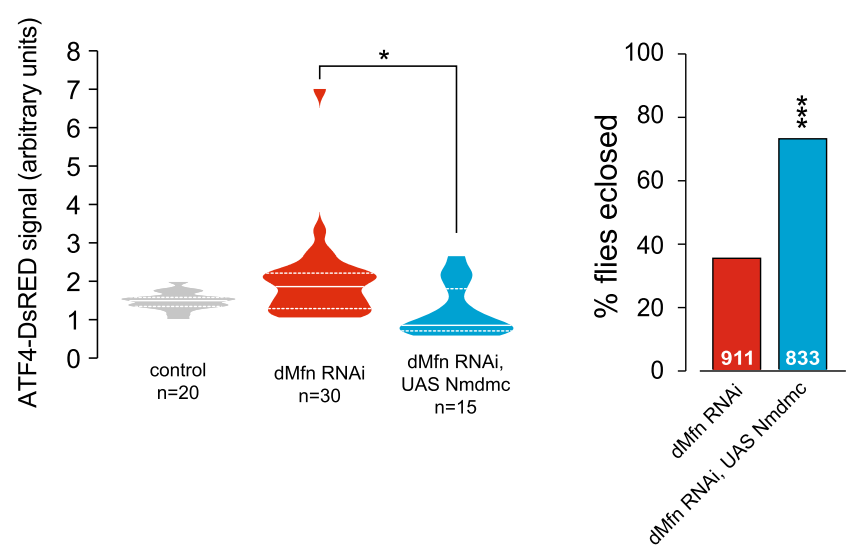

E

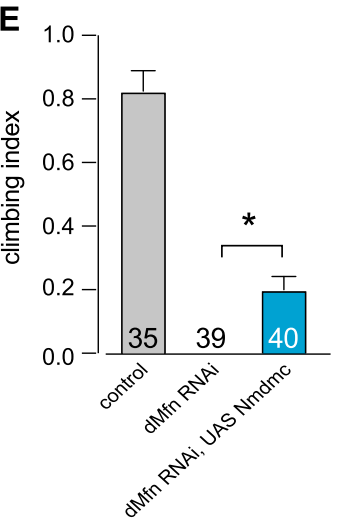

Fig. 7 Expression of $\mathbf{N m d m c}$ rescues defects associated with downregulation of $\boldsymbol{d} M \mathbf{M f n}$. Expression of Nmdmc rescues (a) the loss of $\triangle \psi m$ in dMfn RNAi flies, mean \pm SD; asterisks, one-way ANOVA with Bonferroni's multiple comparison test and (b) the increased expression of $d A T F 4$ in dMfn RNAi flies (asterisks, one-way ANOVA with Dunnett's multiple comparison test). $\mathbf{c}$ Expression of Nmdmc prevents the eclosion defects in dMfn RNAi flies (asterisks, chi-square two-tailed, 95\% confidence intervals). $\mathbf{d}$ Expression of Nmdmc increases the lifespan of $d M f n$ RNAi flies. The $p$-value is shown for the log-rank Mantel-Cox test. e Expression of Nmdmc increases locomotor activity of $d M f n$ RNAi flies. Flies were tested using a standard climbing assay (mean \pm SD; asterisks, one-way ANOVA with Dunnett's multiple comparison test). Genotypes; (a, c-e) Control: w; + ; daGal4/4, dMfn RNAi: w; dMfn RNAi/+; daGal4/+, dMfn RNAi, UAS Nmdmc: w; dMfn RNAi/UAS Nmdmc; daGal4/+, (b) Control: w; ATF4 5'UTR DsRed/+; hhGal4,UASGFP/+, dMfn RNAi: w; ATF4.5'UTR > dsRed/dMfn RNAi; hhGal4,UASGFP/+, dMfn RNAi, UAS Nmdmc: w; ATF4.5'UTR > dsRed/dMfn RNAi, UAS Nmdmc; hhGal4,UASGFP/+. Dataset in A, labelled dMfn RNAi, is also used in Fig. 6b, labelled dMfn RNAi (NF)

milk for $1 \mathrm{~h}$ at room temperature, then probed with the primary antibodies (anti- $\alpha$-tubulin, Sigma, T6074) or antidMfn (a gift from A. Whitworth, MRC, Mitochondrial Biology Unit, University of Cambridge, Cambridge, UK), before being incubated with the appropriate HRPconjugated secondary antibody. Antibody complexes were visualised by Pierce enhanced chemiluminescence (ECL).

\section{Climbing assay}

Climbing assays were performed as previously described $^{22}$ using a counter-current apparatus equipped with six chambers. A total of 15-20 male 3-day-old flies were placed into the first chamber, tapped to the bottom, and then given $20 \mathrm{~s}$ to climb a distance of $10 \mathrm{~cm}$. The flies that successfully climbed $10 \mathrm{~cm}$ or beyond within $20 \mathrm{~s}$ were then shifted to a new chamber, and both sets of flies were given another opportunity to climb the $10 \mathrm{~cm}$ distance. This procedure was repeated a total of five times. After five trials, the number of flies in each chamber was counted. A video demonstrating this technique can be found at https://youtu.be/vmR6s_WAXgc. The climbing index was measured using a weighted average approach with the following formula:

$$
\frac{(0 * n 0)+(1 * n 1)+(2 * n 2)+(3 * n 3)+(4 * n 4)+(5 * n 5)}{5 * \operatorname{SUM}(n 0: n 5)}
$$

In this formula, $n 0$ corresponds to the number of flies that failed the first trial, and $n 1$ through $n 5$ are the numbers of flies that successfully passed each successive trial. At least 100 flies were used for each genotype tested. 


\section{Lifespan analysis}

Groups of 15 newly eclosed males of each genotype were placed into separate vials with food and maintained at $25^{\circ} \mathrm{C}$. The flies were transferred to vials containing fresh food every two to three days, and the number of dead flies was recorded. The data are presented as Kaplan-Meier survival distributions, and the significance was determined by the log-rank test.

\section{Dietary supplements}

Folic acid (Sigma, F7876) was incorporated into the fly food at a final concentration of $4 \mathrm{mM}$. The animals were treated with FA throughout development. Adult flies were maintained on FA-containing food throughout their lifespan, and they were transferred to vials with fresh food every two to three days.

\section{Microscopy-based assessment of mitochondrial function and length}

Measurement of $\Delta \psi \mathrm{m}$ in brains of 3-day-old flies was performed using tetramethylrhodamine (TMRM) as previously described ${ }^{17}$. Briefly, adult fly brains were loaded with $40 \mathrm{nM}$ TMRM in loading buffer (10 mM HEPES, $\mathrm{pH}$ 7.35, $156 \mathrm{mM} \mathrm{NaCl}, 3 \mathrm{mM} \mathrm{KCl}, 2 \mathrm{mM} \mathrm{MgSO}_{4}, 1.25 \mathrm{mM}$ $\mathrm{KH}_{2} \mathrm{PO}_{4}, 2 \mathrm{mM} \mathrm{CaCl}$ and $10 \mathrm{mM}$ glucose) for $40 \mathrm{~min}$ at room temperature, and the dye was present during the experiment. In this experiment, TMRM was used in the redistribution mode to assess $\Delta \psi \mathrm{m}$, and therefore, a reduction in TMRM fluorescence represents mitochondrial depolarisation. Confocal images were obtained using a Zeiss 510 confocal microscope equipped with a $40 \times$ oil immersion objective. Illumination intensity was kept to a minimum (at $0.1-0.2 \%$ of laser output) to avoid phototoxicity, and the pinhole was set to give an optical slice of $2 \mu \mathrm{m}$. Fluorescence was quantified by exciting TMRM using the $565 \mathrm{~nm}$ laser and measured above $580 \mathrm{~nm}$. Zstacks of 5 fields of $300 \mu \mathrm{m}^{2}$ each per brain were acquired, and the mean maximal fluorescence intensity was measured for each group.

Mitochondrial length was quantitated in mechanosensory axons in the ventral nerve cord (VNC) from third-instar larvae. The VNC was dissected in PBS, transferred to a drop of PBS as mounting medium on glass slides, covered with a coverslip and imaged on a Zeiss LSM510 confocal microscope. Mitochondrial length was calculated using the "segmented line" tools in Image to measure the length of mitoGFP-positive mitochondria across their largest dimension. $Z$-projection of $10 \mu \mathrm{m}$-thick stacks was used to follow the mitochondrial 3D distribution and measure lengths more accurately.

\section{Citrate synthase assay}

Citrate synthase activity was measured using a protocol adapted from the Citrate Synthase Assay kit (CS070
SIGMA). Ten male flies (3-days-old) were homogenised in lysis buffer $(100 \mathrm{mM} \mathrm{KCl}, 20 \mathrm{mM}$ Hepes at $\mathrm{pH} 7.5,5 \%$ (v/v) glycerol, $10 \mathrm{mM}$ EDTA, 0.1\% (v/v) Triton X-100, 10 $\mathrm{mM}$ DTT, $1 \mu \mathrm{g} / \mathrm{mL}$ leupeptin, $1 \mu \mathrm{g} / \mathrm{mL}$ antipain, $1 \mu \mathrm{g} / \mathrm{mL}$ chymostatin and $1 \mu \mathrm{g} / \mathrm{mL}$ pepstatin). The suspensions were cleared twice by centrifugation at 2,000 $\times g$ for $15 \mathrm{~s}$ at $4{ }^{\circ} \mathrm{C}$, and the protein concentrations were determined by Bradford assay (Bio-Rad). Sample volume was resuspended in reaction buffer $(75 \mathrm{mM}$ Tris- $\mathrm{HCl} \mathrm{pH} \mathrm{8,} 100 \mu \mathrm{M}$ DTNB, $0.1 \%$ Triton, $350 \mu \mathrm{g} / \mathrm{ml}, 0.5 \mathrm{mM}$ Oxalacetate) and absorbance was measured at $412 \mathrm{~nm}$ for $2 \mathrm{~min}$ using M200PRO plate reader (TECAN, Switzerland). Absorbance values were plotted against time $(\mathrm{min})$ for each reaction. Changes in absorbance $(\Delta \mathrm{A} 412 /$ minute) were used to calculate the citrate synthase activity using the following equation: units $(\mathrm{mmole} / \mathrm{ml} / \mathrm{min})=(\Delta \mathrm{A} 412 /$ $\min \times \mathrm{V}(\mathrm{ml}) / \varepsilon^{\mathrm{mM}} \times \mathrm{L}(\mathrm{cm}) \times \mathrm{V}_{\mathrm{enz}}(\mathrm{ml}) . \mathrm{V}(\mathrm{ml})=$ reaction volume, $\varepsilon^{\mathrm{mM}}=13.6 \mathrm{mM}^{-1} \mathrm{~cm}^{-1}, V_{\text {enz }}(\mathrm{ml})=$ volume of sample. Units of citrate synthase activity were normalised to protein concentration $(\mathrm{mg} / \mathrm{ml})$.

\section{ATP assays}

Five male flies (3-days-old) were homogenised in $100 \mu \mathrm{L}$ of $6 \mathrm{M}$ guanidine- $\mathrm{HCl}$ in extraction buffer $(100 \mathrm{mM}$ Tris and $4 \mathrm{mM}$ EDTA, pH 7.8) to inhibit ATPases. Homogenised samples were subjected to rapid freezing in liquid nitrogen followed by boiling for $5 \mathrm{~min}$. Samples were then cleared by centrifugation and the supernatant was diluted $(1 / 50)$ with extraction buffer and mixed with luminescent solution (CellTiter-Glo Luminescent Cell Viability Assay, Promega). The luminescence was measured on an infinitive M200PRO plate reader (TECAN, Switzerland). The relative ATP levels were calculated by dividing the luminescence by the total protein concentration, which was determined by the Bradford method.

\section{Analysis of ATF4 activation in Drosophila wing discs}

To analyse ATF4 activation, ATF4.5'UTR $>$ dsRed reporter larvae with the expression of GFP in the posterior compartment of wing discs under the control of $h h \mathrm{Gal} 4$ were used. Wing discs were dissected in PBS and imaged on a Zeiss LSM510 confocal microscope. The 15 $\mu \mathrm{m}$-thick stacks were acquired and maximum projection of DsRed signal was measured by using ImageJ software.

\section{Measurement of mitochondrial ROS in Drosophila adult brain}

Drosophila adult brains were dissected in PBS and incubated with $5 \mu \mathrm{M}$ MitoSOX ${ }^{\mathrm{TM}}$ Red mitochondrial superoxide indicator (M36008, Molecular Probes) for 30 min. After incubation brains were washed with PBS for 10 min and imaged on a Zeiss LSM510 confocal microscope. The $100 \mu \mathrm{m}$-thick stacks were acquired and used to measure the MitoSOX signal using ImageJ software. 


\section{Analysis of mitochondrial speed}

Axonal mitochondrial speeds were measured as previously described ${ }^{23,24}$. The 2-5-day-old flies expressing GFP under the $d p r$ driver were anaesthetised with $\mathrm{CO}_{2}$ and enclosed in a custom-built chamber formed by cover glasses where the body of the fly was placed ventral side up and wings were positioned under small coverslips and covered with a drop of halocarbon oil. Imaging of the axonal wing nerve was performed using a Zeiss LSM510 confocal microscope with a $100 \times$ oil immersion objective. Mitochondrial movements were quantified using ImageJ. Zprojection was performed and anterograde and retrograde mitochondrial movements were scored following the direction of movement as previously reported. Anterograde runs towards the fly thorax and retrograde to the cell body of the neuron ${ }^{24}$. Transported mitochondria were manually tracked across the frames and speeds calculated dividing the total distance by the time. Data were plotted using Prism (GraphPad).

\section{Electron microscopy}

For transmission electron microscopy, adult fly brains were fixed overnight in $0.1 \mathrm{M}$ sodium cacodylate buffer ( $\mathrm{pH}$ 7.4) containing 2\% paraformaldehyde, $2.5 \%$ glutaraldehyde and $0.1 \%$ Tween-20. Then, the samples were post-fixed for $1 \mathrm{~h}$ at room temperature in a solution containing $1 \%$ osmium tetroxide and $1 \%$ potassium ferrocyanide. After fixation, the samples were stained en bloc with $5 \%$ aqueous uranyl acetate overnight at room temperature; then, they were dehydrated via a series of ethanol washes and embedded in TAAB epoxy resin (TAAB Laboratories Equipment Ltd., Aldermaston, UK). Semi-thin sections were stained with toluidine blue, and areas of the sections were selected for ultramicrotomy. Ultrathin sections were stained with lead citrate and imaged using a MegaView 3 digital camera and iTEM software (Olympus Soft Imaging Solutions GmbH, Münster, Germany) with a Jeol 100CXII electron microscope (Jeol UK Ltd., Welwyn Garden City, UK).

\section{Microarray acquisition and analysis}

RNA was prepared from 3-day-old male adult flies (8 samples in total, 4 replicates for each genotype). The $d M f n$ RNAi was driven by $d a$ GAL4. The RNA quality was confirmed using an Agilent 2100 Bioanalyzer (Agilent Technologies, CA, USA). Detailed experimental protocols and raw data were deposited in ArrayExpress under accession E-MTAB-6579. Differential expression was analysed using the Partek Genomics Suite (Partek Inc. Missouri, USA). Pathway enrichments were calculated using a pathway ANOVA statistical model in Partek Pathway (Partek Inc. Missouri, USA).

\section{Respirometry}

Mitochondrial respiration in 3-day-old flies was assayed at $37^{\circ} \mathrm{C}$ by high-resolution respirometry as previously described $^{25}$. The OROBOROS Oxygraph DatLab software package (OROBOROS, Innsbruck, Austria) was used for data acquisition (2 s time intervals) and analysis, including calculation of the time derivative of the oxygen concentration and signal deconvolution dependent on the response time of the oxygen sensor, with correction for instrumental background oxygen flux. Respiration was assessed by homogenising two flies using a pestle in MiR05 respiration buffer $(20 \mathrm{mM}$ HEPES, $10 \mathrm{mM}$ $\mathrm{KH}_{2} \mathrm{PO}_{4}, 110 \mathrm{mM}$ sucrose, $20 \mathrm{mM}$ taurine, $60 \mathrm{mM} \mathrm{K}$ lactobionate, $0.5 \mathrm{mM}$ EGTA, $3 \mathrm{mM} \mathrm{MgCl}$, and $1 \mathrm{~g} / \mathrm{l}$ fatty acid-free BSA). Coupled state 3 respiration for complex I was assayed in MiR05 respiration buffer in the presence of $2 \mathrm{mM}$ malate, $10 \mathrm{mM}$ glutamate and $5 \mathrm{mM}$ ADP.

\section{Statistical analyses}

Descriptive and inferential statistical analyses were performed using GraphPad Prism 8 (www.graphpad.com). The data are presented as the mean value, and the error bar indicates \pm SD or \pm SEM (as indicated). In the combined violin and box plots, the median is shown with a solid white line and the quartiles are represented by the dashed white lines. The number of biological replicates per experimental variable $(n)$ is indicated in either the respective figure or figure legend. Significance is indicated as ${ }^{* * * * *}$ for $p<0.0001$; ${ }^{* * * *}$ for $p<0.001$; ** for $p<0.01$ and * for $p<0.05$. The investigators gathering quantitative data on the biological samples were not blinded to the sample identities at the time of analysis. No specific randomisation strategies were employed when the biological replicates were assigned to the treatment groups.

\section{Digital image processing}

Western blot images were acquired as uncompressed, bitmapped digital images (TIFF format). The images were processed using Adobe Photoshop, employing established scientific imaging workflows ${ }^{26}$.

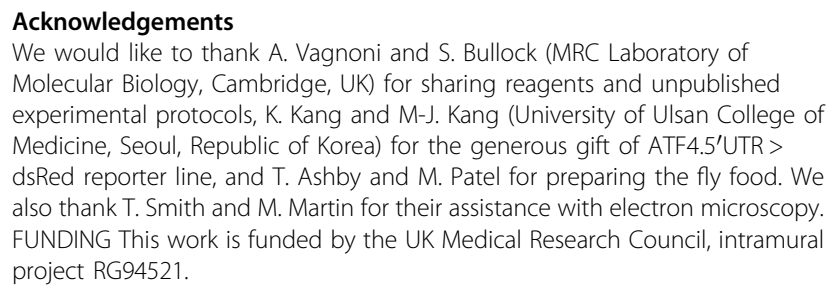
also thank T. Smith and M. Martin for their assistance with electron microscopy. FUNDING This work is funded by the UK Medical Research Council, intramural project RG94521.

\section{Author Contributions}

J.G.M., I.C., A.C.C., S.L., S.H.Y.L. and L.M.M. conceived, designed and performed the experiments and analysed the data. S.H.Y.L. and L.M.M. wrote the paper and contributed equally as joint last authors.

Conflict of interest

The authors declare that they have no conflict of interest. 


\section{Publisher's note}

Springer Nature remains neutral with regard to jurisdictional claims in published maps and institutional affiliations.

Supplementary Information accompanies this paper at (https://doi.org/ 10.1038/s41419-019-1496-2).

Received: 14 December 2018 Accepted: 25 February 2019 Published online: 25 March 2019

\section{References}

1. Skre, H. Genetic and clinical aspects of Charcot-Marie-Tooth's disease. Clin. Genet. 6, 98-118 (1974).

2. Zuchner, S. et al. Mutations in the mitochondrial GTPase mitofusin 2 cause Charcot-Marie-Tooth neuropathy type 2A. Nat. Genet. 36, 449-451 (2004). ng1341 [pii].

3. de Castro, I. P., Martins, L. M. \& Tufi, R. Mitochondrial quality control and neurological disease: an emerging connection. Expert. Rev. Mol. Med. 12, e12 (2010).

4. de Brito, O. M. \& Scorrano, L. Mitofusin 2 tethers endoplasmic reticulum to mitochondria. Nature 456, 605-610 (2008).

5. Celardo, I. et al. Mitofusin-mediated ER stress triggers neurodegeneration in pink1/parkin models of Parkinson's disease. Cell Death Dis. 7, e2271 (2016).

6. Ugur, B., Chen, K. \& Bellen, H. J. Drosophila tools and assays for the study of human diseases. Dis. Model Mech. 9, 235-244 (2016).

7. Lu, B. \& Vogel, H. Drosophila models of neurodegenerative diseases. Annu. Rev. Pathol. 4, 315-342 (2009).

8. Dorn, G. W. 2nd et al. MARF and Opa1 control mitochondrial and cardiac function in Drosophila. Circ. Res. 108, 12-17 (2011).

9. Debattisti, V., Pendin, D., Ziviani, E., Daga, A. \& Scorrano, L. Reduction of endoplasmic reticulum stress attenuates the defects caused by Drosophila mitofusin depletion. J. Cell. Biol. 204, 303-312 (2014).

10. Magwere, T. et al. The effect of dietary restriction on mitochondrial protein density and flight muscle mitochondrial morphology in Drosophila. J. Gerontol. A. Biol. Sci. Med. Sci. 61, 36-47 (2006).

11. Hollenbeck, P. J. The pattern and mechanism of mitochondrial transport in axons. Front. Biosci. 1, d91-d102 (1996).
12. Misko, A. L., Sasaki, Y., Tuck, E., Milbrandt, J. \& Baloh, R. H. Mitofusin2 mutations disrupt axonal mitochondrial positioning and promote axon degeneration. J. Neurosci. 32, 4145-4155 (2012).

13. Celardo, I., Lehmann, S., Costa, A. C. \& Loh, S. H. \& Miguel Martins, L. dATF4 regulation of mitochondrial folate-mediated one-carbon metabolism is neuroprotective. Cell Death Differ. 24, 638-648 (2017).

14. Kang, K., Ryoo, H. D., Park, J.-E., Yoon, J.-H. \& Kang, M.-J. A Drosophila reporter for the translational activation of ATF4 marks stressed cells during development. PLoS One 10, e0126795 (2015).

15. Kilberg, M. S., Shan, J. \& Su, N. ATF4-dependent transcription mediates signaling of amino acid limitation. Trends Endocrinol. Metab. 20, 436-443 (2009).

16. Kelly, G. S. Folates: supplemental forms and therapeutic applications. Altern. Med. Rev. 3, 208-220 (1998).

17. Tufi, R. et al. Enhancing nucleotide metabolism protects against mitochondrial dysfunction and neurodegeneration in a PINK1 model of Parkinson's disease. Nat. Cell Biol. 16, 157-166 (2014).

18. Filadi, R., Pendin, D. \& Pizzo, P. Mitofusin 2: from functions to disease. Cell Death Dis. 9, 330 (2018).

19. Kawalec, M. et al. Mitofusin 2 deficiency affects energy metabolism and mitochondrial biogenesis in MEF cells. PLoS One 10, e0134162 (2015).

20. Rana, A. et al. Promoting Drp1-mediated mitochondrial fission in midlife prolongs healthy lifespan of Drosophila melanogaster. Nat. Commun. 8, 448 (2017).

21. Franco, A. et al. Correcting mitochondrial fusion by manipulating mitofusin conformations. Nature 540, 74-79 (2016).

22. Greene, J. C. et al. Mitochondrial pathology and apoptotic muscle degeneration in Drosophila parkin mutants. Proc. Natl. Acad. Sci. USA 100, 4078-4083 (2003).

23. Vagnoni, A. \& Bullock, S. L. A simple method for imaging axonal transport in aging neurons using the adult Drosophila wing. Nat. Protoc. 11, 1711-1723 (2016).

24. Vagnoni, A., Hoffmann, P. C. \& Bullock, S. L. Reducing Lissencephaly-1 levels augments mitochondrial transport and has a protective effect in adult Drosophila neurons. J. Cell. Sci. 129, 178-190 (2016).

25. Costa, A., Loh, S. \& Martins, L. M. Drosophila Trap1 protects against mitochondrial dysfunction in a PINK1/parkin model of Parkinson's disease. Cell Death Dis. 4, e467 (2013).

26. Wexler, E. J. Photoshop CS3 Extended for Biomedical Research [DVD-ROM and online course]. (Lynda.com, Ventura, 2008). 